\title{
Effect of Incident Laser Sheet Orientation on the OH-PLIF Imaging of Detonations
}

\author{
K.P. Chatelain • R. Mével • J. Melguizo-Gavilanes • A. Chinnayya • S. \\ $\mathrm{Xu}$. D.A. Lacoste
}

Received: date / Accepted: date

\begin{abstract}
This study aims to investigate the effect of laser sheet orientation on the OH-PLIF imaging of a detonation wave and to identify the potential benefit of using a transverse laser orientation, compared to the conventional frontal orientation. In this study, we developed a new LIF model, based on a pre-existing one, to include a more fundamental calculation of the absorption lineshape and the effects of the laser orientation on the fluorescence signal. One- and two-dimensional simulations are used to validate this new LIF model for both laser orientations using OH-PLIF images from the literature. The effect of the two laser orientations on detonation imaging is investigated numerically on a
\end{abstract}

K.P. Chatelain · D.A. Lacoste

King Abdullah University of Science and Technology (KAUST), Clean Combustion Research Center (CCRC), Thuwal, Saudi Arabia,

Tel.: +966128084783

E-mail:

karl.chatelain@kaust.edu.sa,

deanna.lacoste@kaust.edu.sa

ORCID id: 0000-0003-4902-3337, 0000-0002-4160-4762

R. Mével

Center for Combustion Energy, Tsinghua University, Beijing,

China

Department of Automotive Engineering, Tsinghua University, Beijing, China,

E-mail: mevel@mail.tsinghua.edu.cn

ORCID id: 0000-0002-0032-350X

J. Melguizo-Gavilanes · A. Chinnayya

Institut Pprime, UPR 3346, CNRS, ENSMA, University of

Poitiers, Futuroscope-Chasseneuil, France,

E-mail: josue.melguizo-gavilanes@cnrs.pprime.fr, E-mail: ashwin@ensma.fr

ORCID id: 0000-0001-5174-6003, 0000-0002-8728-2148

S. Xu

School of Aerospace Engineering, Tsinghua University, Beijing, China,

E-mail: slxu@tsinghua.edu.cn
2D $\mathrm{OH}$ field. Based on these results, the frontal orientation seems more suitable to collect information near the detonation front, while the transverse laser orientation can give more quantitative information far from the front and only for short optical paths. With the present laser configuration and experimental conditions, both laser orientations present the same limitation with a strong laser energy absorption for long optical paths. While these first results show a qualitative agreement with experimental data, new experimental data are required to quantitatively validate this promising transverse laser orientation and confirm its relevance for $\mathrm{OH}-$ PLIF imaging of detonations.

Keywords detonation front visualization · OH-planar laser induced fluorescence (OH-PLIF) - spectroscopic modeling · LIF model

\section{Introduction}

Planar Laser-Induced Fluorescence of $\mathrm{OH}$ radical $(\mathrm{OH}-$ PLIF) is a powerful technique used to characterize the structure of the reaction zone during the propagation of weakly $[1,2]$ and highly unstable detonations [3,4]. This technique has also been employed to characterize the reinitiation mechanism of a highly unstable detonation in a bifurcation channel $[5,6]$. In weakly unstable detonations, the regular and smooth shock front and the related gas dynamics result in a well defined and regular keystone-like structure of the reaction zone. The combustion is essentially driven by the shock-induced autoignition mechanism. In highly unstable detonations, the keystone shape of the reaction zone, although still visible, appears much less sharp. The reaction front is wrinkled and demonstrates small-scale variations in intensity. Pockets of low fluorescence intensity are visible 
behind the detonation front. The combustion is then driven by both shock-induced ignition and diffusioncontrolled burning [7].

To enable direct comparison between the experimental PLIF images and 2D numerical simulations, Mével et al. $[8,9]$ developed a LIF model to obtain numerical PLIF images. They showed that the PLIF images become increasingly less representative of the $\mathrm{OH}$ field further behind the shock front. Under most detonation conditions, the LIF process is within the optically-thick regime due to the high $\mathrm{OH}$ concentrations. In other words, this optically-thick regime corresponds to the strong absorption of the incident laser light by the high $\mathrm{OH}$ concentration at the beginning of the reaction zone, which makes the zones of high $\mathrm{OH}$ concentration (away from the front) less visible due to the attenuation of the laser light as it travels through the medium. Such an effect was also highlighted by Mével under shock tube/pulsed laser-induced fluorescence conditions [10]. In [10], three regimes were identified depending on the characteristics of the optical path: (i) optically thin; (ii) optically thick; and (iii) non-physical. Although the study of Mével et al. [8] examined in detail the effect of various parameters on the PLIF intensity, they have focused on a single laser orientation, the frontal, for which the laser sheet propagation is opposed to the detonation wave propagation. However, other studies [11-13] employed a different experimental configuration, the transverse, for which the laser sheet propagation is perpendicular to the detonation wave propagation. This orientation might enable observation of regions of high $\mathrm{OH}-$ concentration far downstream of the detonation front. The goal of the present study is to investigate the effect of the incident laser light sheet orientation on the OH-PLIF imaging of a detonation wave reaction zone. For the purpose of this contribution, we limited our investigation to a weakly unstable detonation wave in hydrogen-oxygen mixtures highly diluted with argon to enable direct comparison with the experimental $\mathrm{OH}-$ PLIF images available in the literature. An updated LIF model was developed and validated using steady one-dimensional (1D) and unsteady two-dimensional (2D) numerical simulations. A comparison of two numerical OH-PLIF images obtained with the aforementioned laser orientations is performed to highlight the benefits and drawbacks of the two laser orientations.

\section{Numerical simulation methods}

\subsection{Laser induced fluorescence model}

Similar to Mével et al. [8], we employed the 3-level LIF model of Bessler et al. [14]. In the linear regime and at steady-state, the fluorescence intensity for one pumped electronic transition is given by Eq. 1,

$F \propto f_{B} \Gamma I_{\nu}^{0} I_{b} N_{O H} B \frac{1}{Q} \sum A_{i}$,

where $f_{B}$ is the Boltzmann fraction, $\Gamma$ is the dimensionless overlap integral, $I_{\nu}^{0}$ is the normalized spectral laser irradiance, $I_{b}$ is a dimensionless factor accounting for the light sheet absorption, $N_{O H}$ is the $\mathrm{OH}$ radical number density, $B$ is the Einstein B coefficient, $Q$ is the quenching rate, and $A_{i}$ are the Einstein A coefficients.

The parameters in Eq. 1 are calculated as described in Mével et al. [8] with five main changes: (i) different laser sheet orientations with respect to the detonation front can now be considered; (ii) the collisional broadening $\left(\Delta \nu_{c}\right)$ is calculated (more accurately) using Eq. 2,

$\Delta \nu_{c}=P \sum_{A} \mathrm{X}_{A} \sigma_{A B}^{2}\left(\frac{8}{\pi \mu_{A B} k T}\right)^{1 / 2}$

where $P$ is the pressure, $T$ is the temperature, $k$ is the Boltzmann's constant, $\mathrm{X}_{A}$ is the mole fraction of species $A$, and $\sigma_{A B}$ and $\mu_{A B}$ correspond, respectively, to the optical collision diameter and the reduced mass between species $A$ with the fluorescent species $(B=O H)$; (iii) the absorption lineshape function is calculated using a Voigt profile as described in [15]; (iv) when the shifts apply (see section 3.2), the linecenter shift of the absorption lines is calculated considering both the pressure $\left(\delta_{P}\right)$ and the Doppler shift $\left(\delta_{D}\right)$, as described in $[15$, $16]$. For any absorption line $i$, the pressure shifted absorption line is calculated using Eq. 3 and the pressure shift is obtained $\delta_{\mathrm{P}_{i}}=\nu_{i \text {, shifted }}-\nu_{i, 0}$,

$\nu_{i, \text { shifted }}=\nu_{i, 0}+P \sum_{A} \mathrm{X}_{A} \delta_{A, T_{\text {ref }}}\left(\frac{T_{\text {ref }}}{T}\right)^{M}$

where $\nu_{i, 0}$ and $\nu_{i \text {,shifted }}$ correspond to the initial and the shifted absorption linecenter, respectively, for a specific mixture in given temperature and pressure conditions; $\delta_{A, T_{\text {ref }}}$ and $M$ are the pressure shift parameters associated to a given species $A$ (at a given mole fraction $\mathrm{X}_{A}$ ) at a reference temperature $\left(T_{\text {ref }}\right)$. The Doppler shift $\delta_{\mathrm{D}}$ is calculated using Eq. 4,

$\frac{\delta_{\mathrm{D}}}{\nu_{0}}=\frac{u}{c}$

where $\delta_{\mathrm{D}}$ and $\nu_{0}$ are respectively the shift value and the initial absorption linecenter, $u$ and $c$ are respectively the gas mean velocity in the laser direction and the speed of light. The updated LIF model also (v) dynamically considers the contribution of the absorption lines spectrally close to the laser linecenter $( \pm 0.25 \mathrm{~nm})$. The 
importance of these adjacent lines is strongly dependent on the experimental conditions and the position of the laser linecenter. Here, five transitions are considered $\mathrm{P}_{1}(5), \mathrm{Q}_{21}(9), \mathrm{Q}_{2}(8), \mathrm{Q}_{1}(9), \mathrm{Q}_{12}(8)$ (shown in dashed lines in Fig. 1) whereas Mével et al. [8] used only $\mathrm{Q}_{2}(8), \mathrm{Q}_{1}(9)$. This more complex approach is justified by the higher initial pressure used by Wang et al. [13] than that of Austin et al. [4]. The contribution of the adjacent absorption lines is illustrated in Fig. 1 for conditions corresponding to the von Neumann (vN) and the Chapman-Jouguet (CJ) states. At the von Neumann state, the calculated absorption lineshape is $16 \%$ higher, at the laser linecenter, when five lines are considered instead of two. This difference reduces to $4 \%$ at the CJ state. A more detailed evolution of the Voigt profile along the detonation front is presented in Appendix 1.

The dimensions of the laser sheet and the Gaussian spatial distribution of the laser energy [17] are taken into account to accurately compare the experimental and simulated LIF signals for each validation case. Additionally, the curvature of the detonation front is corrected to consider the non-homogeneous optical path in the 1D simulations (ZND). This correction reconstructs the (vertical) optical path, considering a pile of ZND calculations that have the same (vertical) thickness as observed experimentally, to obtain the correct laser absorption. For each ZND calculation, the detonation front is shifted from each other by the offset observed in the experimental image. This correction cannot be applied on the $2 \mathrm{D}$ validation, as the curvature is determined by the simulation.

\subsection{Reaction Model}

The reaction model employed for all the simulations is the reaction model of Mével et al. [18]. It is composed of 9 chemical species (including Ar) and 29 reversible reactions. It has been extensively validated for different hydrogen-oxidizer mixtures and has demonstrated good predictive capabilities for a wide range of experimental conditions including detonation relevant conditions [19-21].

\subsection{Detonation models}

The steady 1D detonation simulations are performed using a ZND code implemented in CHEMKIN-II. It is based on the work of Shepherd [22] and is equivalent to the ZND code available within the Shock and Detonation Toolbox implemented with Cantera [23]. Examples

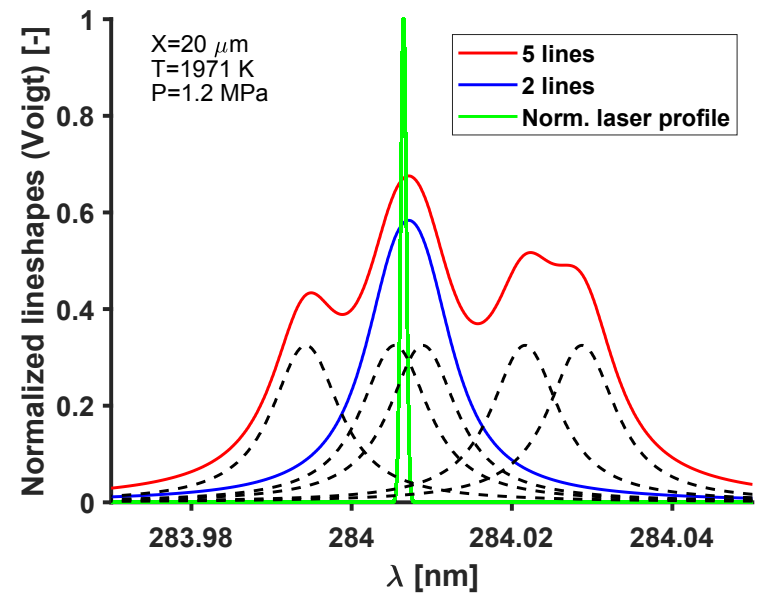

a) von Neumann state

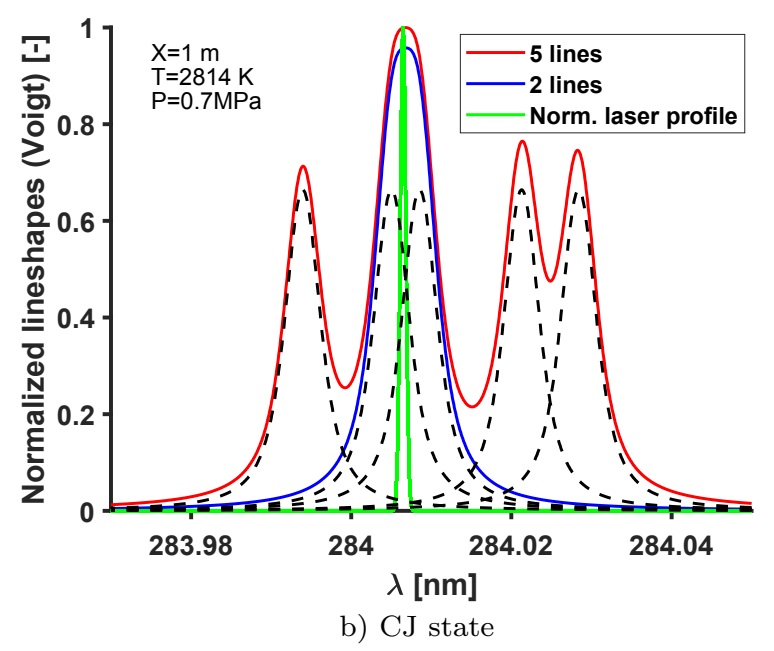

Fig. 1 Effect of the number of absorption lines considered to calculate the absorption lineshapes at two positions $(X)$ behind the detonation leading shock. The normalization is performed considering the maximum of the two Voigt profiles (i.e. the 2-line and the 5-line profiles) over the entire ZND profile. The five absorption lines, represented by black-dashed lines, use the same normalization value. Conditions: $2 \mathrm{H}_{2}-\mathrm{O}_{2}-$ $10 \mathrm{Ar}$ at $P_{1}=45.3 \mathrm{kPa}$ and $T_{1}=295 \mathrm{~K}$

of application of this ZND code can be found in $[20,24$, $25]$.

The unsteady 2D simulations are performed using an inviscid formulation with detailed chemistry with an in-house code RESIDENT (REcycling mesh Simulation of DEtonations). Particulars about the numerical methods used, spatial and temporal discretizations as well as the parallelization methodology can be found in $[26,27]$. Briefly, we use operator splitting to couple the hydrodynamics to the chemistry together with directional splitting, along with a ninth-order monotonicity preserving interpolation in space, and a third order explicit RungeKutta in time. The integration of the chemical source 
terms follows the methodology described in [28] to avoid being limited by the smallest time scales dictated by the chemistry. Although this method was proposed in the context of turbulent simulations, it seems to perform efficiently for detonations as well. Simulations are conducted in the laboratory frame using 400 processors and a fixed squared domain of $40 \mathrm{~mm}$. A sliding window technique [29] is used to keep the propagating detonation within the computational domain at all times. A uniform mesh resolution of $\Delta_{x}=\Delta_{y}=10 \mu \mathrm{m}$ is used in the 2D simulations, which corresponds to a ratio of $\Delta_{i} / \Delta_{x} \sim 20$ [30], where $\Delta_{i}$ is the induction zone length, defined as the distance from the leading shock to maximum thermicity. Increasing the resolution did not result in changes in the numerically predicted cell size.

\subsection{Validation methodology}

To compare the experimental results with the steady 1D simulations, 1D LIF intensity profiles are extracted from the experimental PLIF images. The post-processing sequence is illustrated in Fig. 2 and comprises: (i) defining subdomains of interest based on the detonation front structure; (ii) normalizing the profile in this subdomain; (iii) performing a vertical averaging of the signal to get the 1D profile. These experimental 1D fluorescence profiles are compared with the ZND OH profile and the simulated fluorescence intensity profile.

The $2 \mathrm{D}$ validation of the model relies on a comparison of the experimental images and a $2 \mathrm{D}$ synthetic PLIF image following the methodology described in Mével et al. [8]. As performed with the $1 \mathrm{D}$ validation, this synthetic PLIF image is calculated from the $2 \mathrm{D}$ field of $\mathrm{OH}$ using the LIF model.

\section{Results and discussion}

\subsection{Validation using steady 1D simulation}

\section{Frontal laser orientation}

Figure 3 presents two validation cases of the present LIF model with the experimental results from Austin [1] that uses a frontal laser orientation. The present LIF model reproduces the experimental 1D profiles for all the areas investigated. From this comparison and the results obtained with other areas not presented here, it can be concluded that the modifications made to the LIF model does not degrade the overall agreement between the experimental and calculated profiles for the frontal orientation, obtained previously [8].
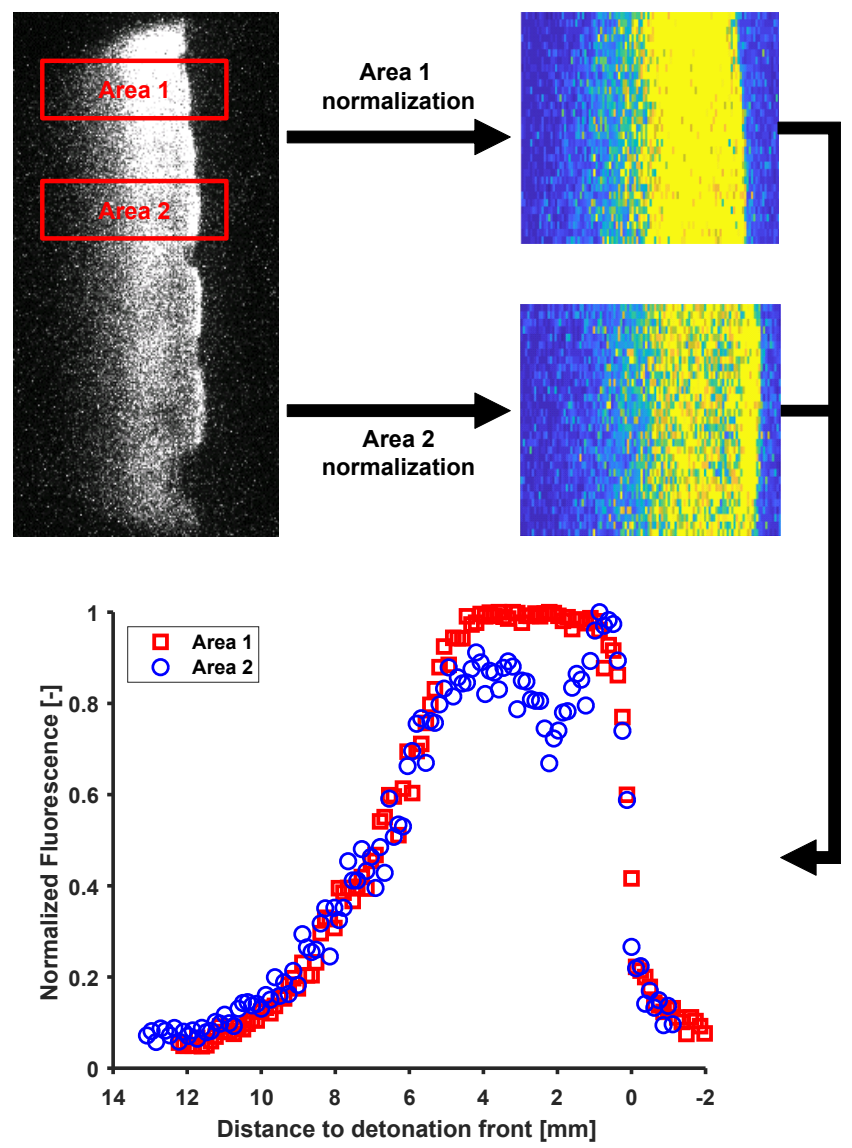

Fig. 2 Post-processing methodology: extraction of 1D LIF intensity profiles from the experimental PLIF images. Conditions: $2 \mathrm{H}_{2}-\mathrm{O}_{2}-10 \mathrm{Ar}$ at $P_{1}=45.3 \mathrm{kPa}$ and $T_{1}=295 \mathrm{~K}$

\section{Transverse laser orientation}

Figure 4 presents two validation cases of the present LIF model with the experimental results from Wang [13], which used a transverse laser orientation. The two areas selected aim to represent conditions for short and long optical path (vertically), respectively.

The experimental 1D LIF signal obtained with the transverse laser orientation is not representative of the $X_{\mathrm{OH}}$ profile and the LIF signal decreases rapidly after the detonation front, similar to the frontal orientation. This decrease seems induced by the experimental setup, that uses a relatively narrow laser sheet $(24 \mathrm{~mm})$ for which only half of the sheet illuminates the reaction front. The other half of the sheet travels through the fresh gases located ahead of the detonation front.

The base numerical LIF signal (referred to as Model with a solid red line in Figs. 4b and 4c) does not seem to be able to reproduce the overall experimental shape. Eventhough these results include the following effects: (i) the non-homogeneous optical path, (ii) the spatial distribution of the laser energy, and (iii) the curvature 


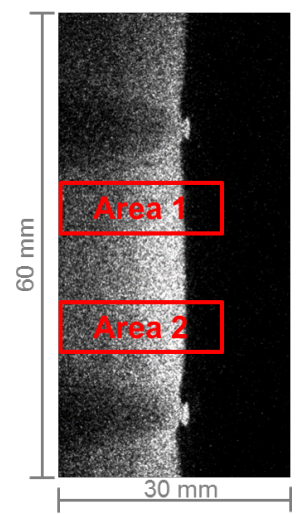

a) Experimental OH-PLIF

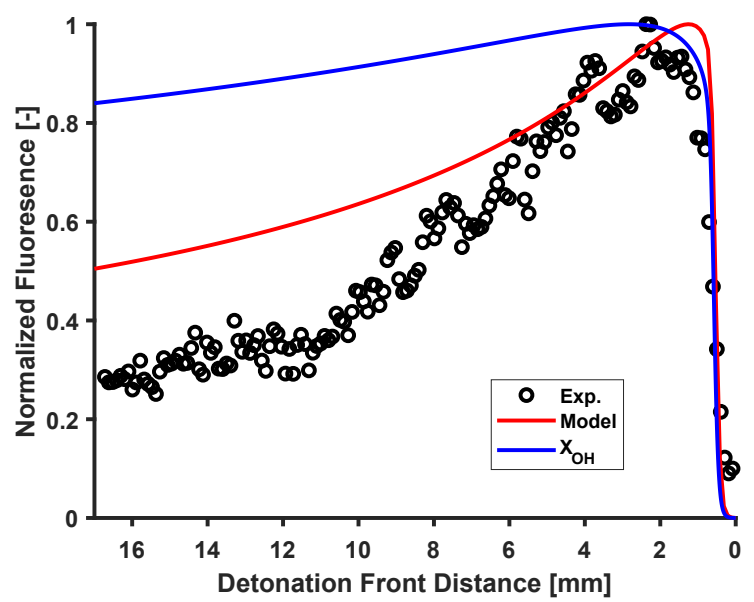

b) Validation on area 1

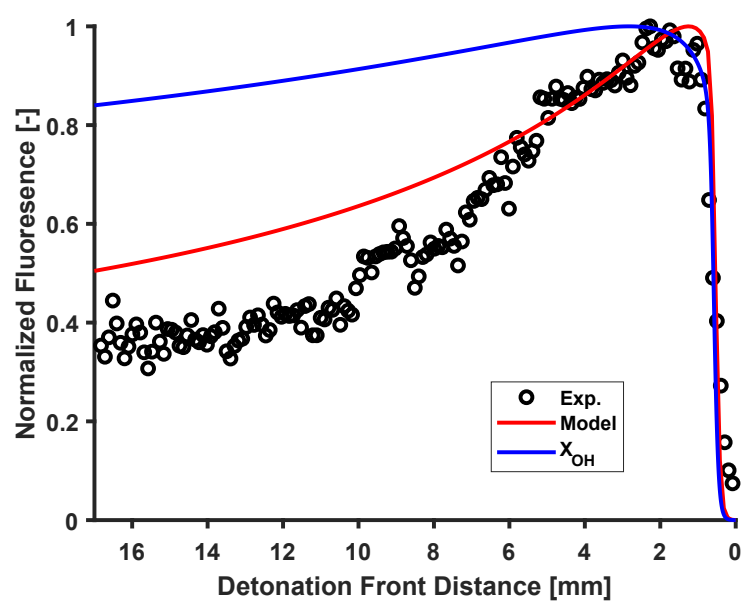

c) Validation on area 2

Fig. 3 1D validation of the frontal laser orientation (from the right) using an OH-PLIF image from Austin [1] in a). 1D experimental (black dots) and ZND (red lines) LIF profiles, obtained for areas 1 and 2 , are respectively presented in b) and c). ZND $\mathrm{OH}$ mole fractions (blue lines) are represented for comparison. Conditions: $2 \mathrm{H}_{2}-\mathrm{O}_{2}-12 \mathrm{Ar}$ at $P_{1}=20 \mathrm{kPa}$ and $T_{1}=295 \mathrm{~K}$

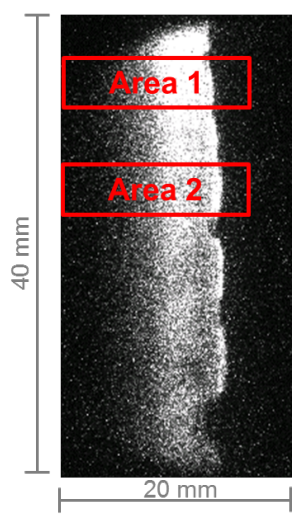

a) Experimental OH-PLIF

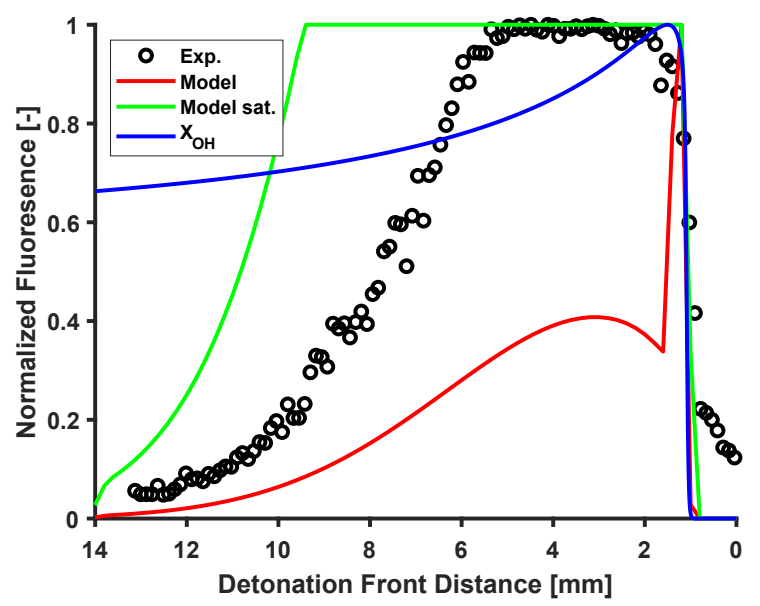

b) Validation on area 1

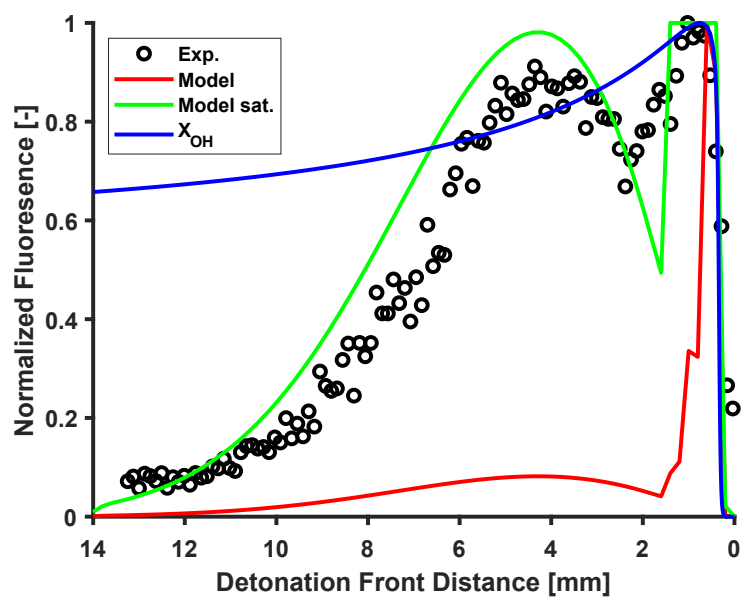

c) Validation on area 2

Fig. 4 1D validation of the transverse laser orientation (from the top) using an OH-PLIF image from Wang [13] in a). 1D experimental (black dots) and ZND (red and green lines) LIF profiles, obtained for areas 1 and 2, are respectively in b) and c). ZND $\mathrm{OH}$ mole fractions (blue lines) are represented for comparison. Conditions: $2 \mathrm{H}_{2}-\mathrm{O}_{2}-10 \mathrm{Ar}$ at $P_{1}=45.3 \mathrm{kPa}$ and $T_{1}=295 \mathrm{~K}$ 
of the detonation front (by shifting the position of the detonation front), the saturation of the experimental images has been neglected. To mimic experimental observations, this saturation is simulated (referred to as Model sat. with a green solid line) by amplifying the LIF signal and clipping the maximum value to 1 . The agreement between the experimental and the modeled LIF signals (Figs. 4b and 4c) is significantly improved by accounting for the saturation, notably the double peak is well reproduced in c). Additional validation cases present similar features and are presented in Appendix 2 .

Observed in most of the area with long (vertical) optical paths, the presence of this double peak is mainly due to the curvature of the detonation and the vertical laser orientation, which produces a non-monotonic energy profile distribution along the detonation front axis (horizontal). The 1D fluorescence profile for area 2 in Fig. 4c can be characterized by three regions: (i) for a short distance behind the leading shock $(\leq 1 \mathrm{~mm})$, the fluorescence signal is at its maximum value, which is due to the high laser intensity reaching this location, as the laser sheet travels through fresh gases only (located vertically above area 2); (ii) the second fluorescence peak (near $5 \mathrm{~mm}$ ) is smaller than the first one, due to the attenuated laser energy received by the $\mathrm{OH}$ molecules at this specific (horizontal) distance behind the leading shock. The double peak shape seems more pronounced in the present conditions due to the narrow laser sheet, which induces a strong fluorescence decay beyond $6 \mathrm{~mm}$ behind the leading shock. (iii) the local fluorescence minimum (near $2 \mathrm{~mm}$ ), located between the two fluorescence peaks, is due to a lower laser energy at this specific location, compared to both (i) and (ii). This lower laser energy is due to the high $\mathrm{OH}$ density pockets in the previous areas located above vertically (or for shorter optical paths).

It is noted that part of the difference of width between the experimental and the simulated signals for area 1 is due to the edge of the optical windows (which is noticeable with the rounded shape at the top of Fig. 4a), which obscures the experimental signal at this location.

Many aspects of the imaging system had to be included in the modeling to obtain qualitative agreement with the experimental data. To the best of our knowledge, all the images currently available in the literature $[11,12]$ obtained with the transverse laser orientation show some degree of saturation, hence no further validation can be performed. Since some of these aspects are difficult to quantify in the present validation data, additional experimental data are required to better validate the model. New experimental data must be obtained with OH-PLIF images free of saturation and with a known spatial distribution of the incident laser energy.

\subsection{Effect of the absorption line shift}

Due to the high pressure and the high flow velocity involved in detonations, both the pressure and the Doppler line shifts, denoted $\delta_{\mathrm{P}}$ and $\delta_{\mathrm{D}}$, are expected to be nonnegligible. This section aims to quantify these values along the detonation front and to identify their relevance to reproduce the LIF signals. Only Austin's conditions can be used to investigate these effects, as $\delta_{\mathrm{D}}=$ 0 for the transverse laser orientation.

Figure 5a presents the evolution of both shifts and the total linecenter shift $\delta_{\text {Tot. }}$ along the detonation for a single absorption line $\left(\mathrm{Q}_{12}(8)\right)$. Similar trends are obtained with the other absorption lines. Although the Doppler line shift $\delta_{\mathrm{D}}$ is important in such high speed flow, with a relative contribution from 80 to $85 \%$ along the detonation, the $\delta_{\text {Tot. }}$ remains low at this condition $\left(\delta_{\text {Tot. }}<1.5 \mathrm{pm}\right)$, as presented in Fig. 5a. In Fig. 5b, the experimental 1D LIF profile is compared with three different simulated LIF profiles. In the first one (Model no shift) none of the absorption line shifts are considered; in the second one (Model $\left.+\delta_{\mathrm{P}}\right)$, only the pressure shift is included; while in the third one (Model $\left.+\delta_{\mathrm{P}+\mathrm{D}}\right)$ both (the Doppler and the pressure shifts) are included. For this condition, the pressure and the Doppler shift have a negligible and limited (a $3.5 \%$ increase of the fluorescence signal in average) effect on the overall fluorescence signal, respectively. As observed in Fig. 5a, the low $\delta_{\text {Tot. }}$ is mainly due to the low pressure conditions used in the validation cases from Austin [1]. However, similar conclusions $\left(\delta_{\text {Tot. }}<1 \mathrm{pm}\right)$ can be drawn for the conditions from Wang [13], obtained at higher initial pressure $\left(P_{1}=45 \mathrm{kPa}\right)$ but with a transverse laser orientation, for which $\delta_{\mathrm{D}}=0$ and $\delta_{\mathrm{P}}<1 \mathrm{pm}$. Further discussions on the absorption line changes as a function of the experimental conditions are presented in Appendix 3.

As presented in Eq. 4, the $\delta_{\mathrm{D}}$ must be calculated with care as it can be either zero (transverse laser orientation), have the same sign as the $\delta_{\mathrm{P}}$ (frontal orientation, meaning laser light propagating opposite to the flow), have the opposite sign as that of $\delta_{\mathrm{P}}$ which can induce a shift to the UV in specific conditions (dorsal laser orientation, meaning laser light propagating in the same direction as the flow). 


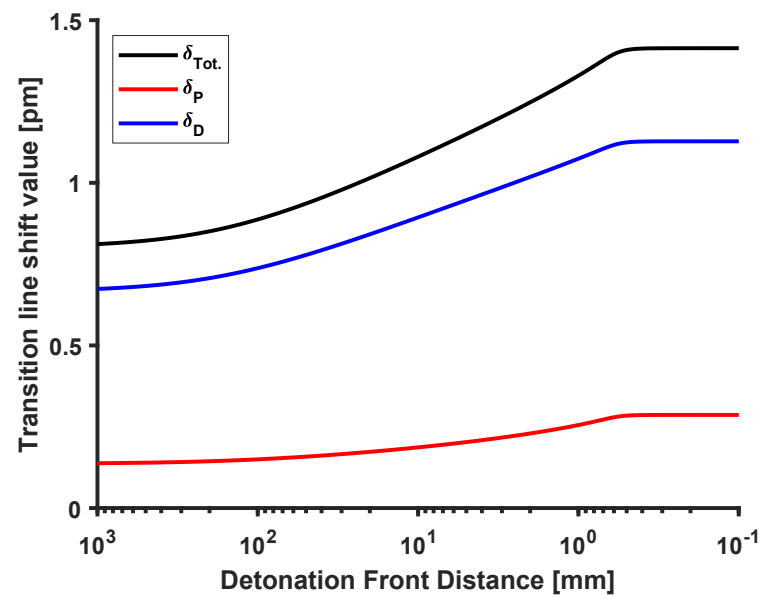

a) $1 \mathrm{D}$ evolution of the absorption line shifts

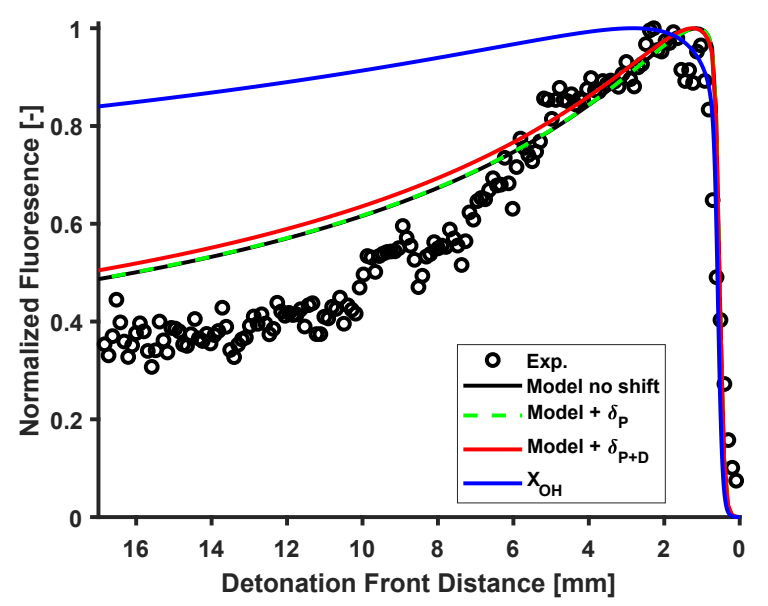

b) Effect of the absorption line shifts on fluorescence

Fig. 5 Quantification of the absorption line shift on a single transition $\left(\mathrm{Q}_{12}(8)\right)$, in a). Effect of the absorption line shifts on the fluorescence signal, in b). The area 2 used previously in the $1 \mathrm{D}$ validation (Fig. 3c) is shown for comparison. Conditions use a frontal laser orientation and a $2 \mathrm{H}_{2}-\mathrm{O}_{2}-12 \mathrm{Ar}$ mixture at $P_{1}=20 \mathrm{kPa}$ and $T_{1}=295 \mathrm{~K}$

\subsection{Unsteady 2D simulation}

Experimental-numerical comparison of the 2D PLIF signals

Figure 6 compares the curved and saturated experimental OH-PLIF field (in a)) with the ideal, essentially flat numerical ones obtained with (in b)) and without corrections (in c)) to consider saturation of the camera. The level of saturation is the same as the one used in the $1 \mathrm{D}$ validation in Fig. 4. As seen in Fig. 6b, the corrected fluorescence signal reproduces qualitatively the experimental OH-PLIF images, notably the width of the front illuminated with the laser sheet and the relative fluorescence intensity between the top and the bottom of the image. As observed in the $1 \mathrm{D}$ validation of the trans- verse orientation, the shape of the front is different on the top left of the images (short optical path). This difference is due to the edge of the visualization window, which is displayed in dashed black lines in Fig. 6b but not considered in the LIF intensity calculation. The numerical OH-PLIF image, in Fig. 6b, qualitatively reproduces the experimental OH-PLIF image from Wang, when both the saturation of the camera and the edge of the visualisation window are accounted for.

Based on the different validation of the LIF model, Fig. 6c aims to represent the hypothetical experimental OH-PLIF image without saturation of the camera. This synthetic OH-PLIF image demonstrates a strong absorption of the laser energy along the vertical axis, which is barely noticeable in the experimental images due to the saturation of the camera. The numerical LIF signal rapidly drops close to 0 after only $20 \mathrm{~mm}$ (from the top of the image). The double peaks observed experimentally, which were reproduced with the 1D simulations (Fig. 4, are also observed on the 2D numerical PLIF (see line at $3.3 \mathrm{~mm}$ in Fig. 7. In addition, even more complex profiles can be observed for different vertical positions (see line at $9.9 \mathrm{~mm}$ in Fig. 7. These more complex profiles are due to the combination of the $\mathrm{OH}$ concentration nonuniformity and the spatial distribution of the laser energy inside the reaction front. These very complex LIF intensity profiles emphasize the need for new experimental data with precise measurements of the laser energy profile to fully validate the transverse orientation of the LIF model.

Effects of the laser orientation the detonation front visualization

The effect of the two laser orientations is investigated using the results from the unsteady $2 \mathrm{D}$ simulation, in Fig. 8. These results consider an ideal laser sheet (without a Gaussian spatial energy distribution) to preclude the effect of the laser energy profile. For a frontal orientation, the present results are similar to those obtained by Mével et al. [8] with a high fluorescence intensity confined to the detonation front. The use of a transverse laser orientation enables to visualize regions of high LIF intensity (in Fig. 8c) far behind the detonation front but limited to $1 \mathrm{~cm}$ vertically. In the case of the transverse laser orientation, the LIF intensity (at least for short optical paths) better represents the $\mathrm{OH}$ profile along the detonation front compared to the frontal laser orientation. In addition, the LIF signal obtained from the transverse laser orientation enables to observe, to some extent, the complex patterns associated with the $\mathrm{OH}$ concentration in Fig. 8a but with different intensities. These observations are not possible from the 


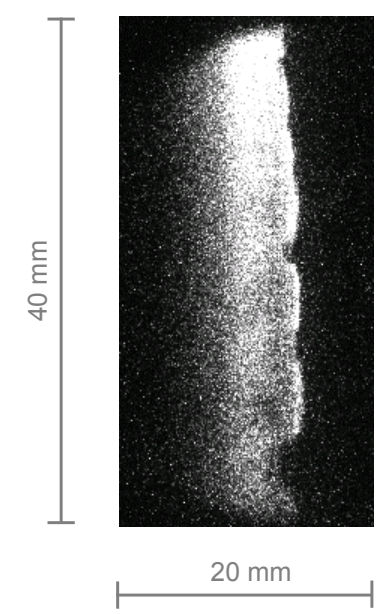

a) Experimental OH-PLIF

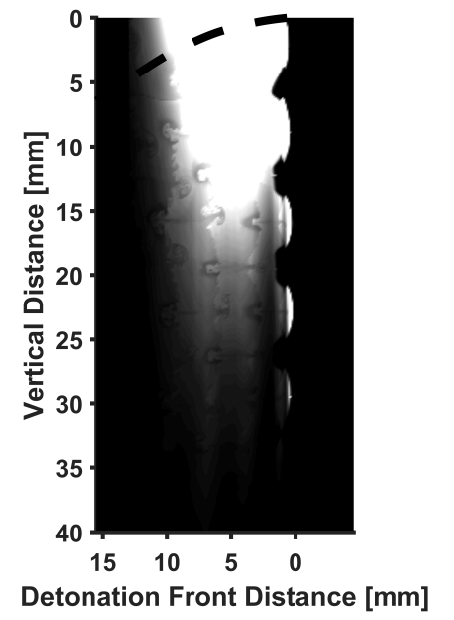

b) Numerical OH-PLIF (corrected)

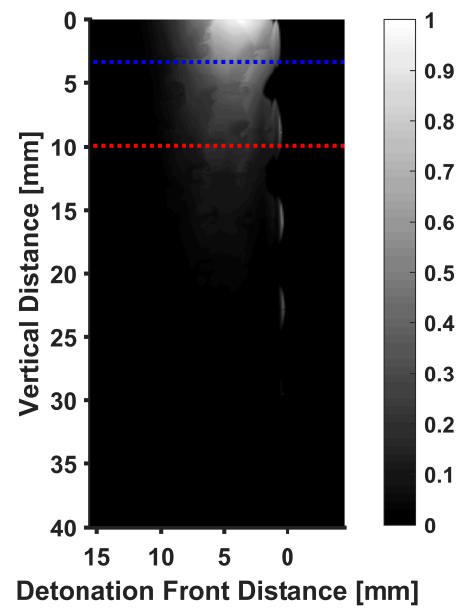

c) Numerical OH-PLIF (no corrections)

Fig. 6 Comparison between experimental and numerical OH-PLIF images. a) experimental OH-PLIF from Wang [13]; b) numerical OH-PLIF including corrections for camera saturation; c) numerical OH-PLIF without corrections for camera saturation. Color scale is identical between b) and c). In b), the dashed line represents the edge of the observation window, based on the experimental dimensions. In c), the blue and red dotted lines represent the location of the two profiles represented in Fig. 7. Conditions: $2 \mathrm{H}_{2}-\mathrm{O}_{2}-10 \mathrm{Ar}$ at $\mathrm{P}_{1}=45.3 \mathrm{kPa}$ and $\mathrm{T}_{1}=295 \mathrm{~K}$

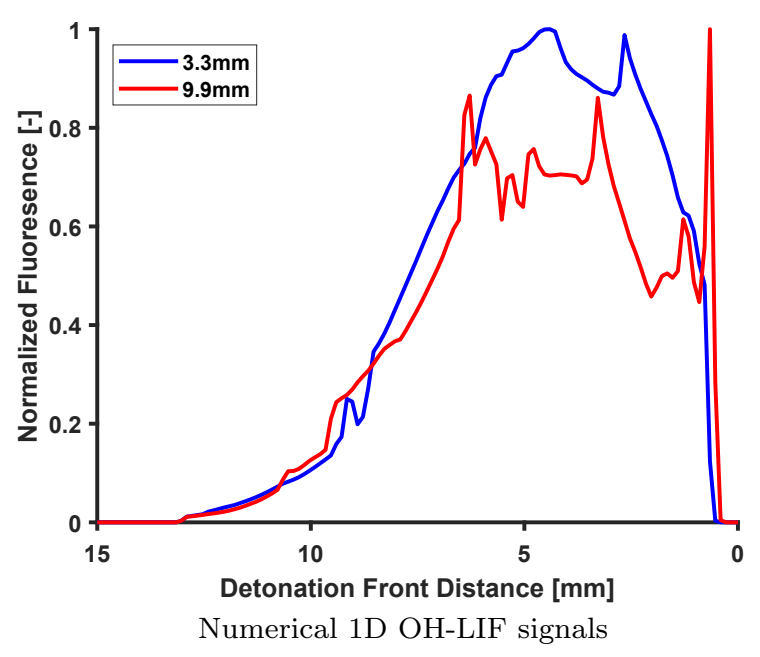

Fig. 7 Normalized 1D LIF profiles extracted at two different heights from Fig. 6c, respectively at $3.3 \mathrm{~mm}$ and $9.9 \mathrm{~mm}$ from the laser entrance (top of the image). Conditions: $2 \mathrm{H}_{2}-\mathrm{O}_{2}-$ $10 \mathrm{Ar}$ at $P_{1}=45.3 \mathrm{kPa}$ and $T_{1}=295 \mathrm{~K}$

frontal laser orientation due to the optical thickness of the detonation front.

\section{Conclusions}

An improved version of the LIF model presented in Mével et al. [8] has been developed and validated against $1 \mathrm{D}$ and $2 \mathrm{D}$ simulations using OH-PLIF images from the literature. Although detonation involves both high speed flow and high pressure variations, the absorption linecenter shifts have a negligible effect on the overall fluorescence signal for the present experimental conditions. The effect of the laser sheet orientation on the $\mathrm{OH}-\mathrm{PLIF}$ imaging is investigated numerically by using the validated LIF model on $2 \mathrm{D} \mathrm{OH}$ concentration distributions obtained from numerical simulations. Based on the present results, the transverse orientation of the incident laser sheet could enable to observe regions of high $\mathrm{OH}$-concentration behind the detonation front. While the visualization of domains with relatively short optical paths enables such observation, domains far from it are significantly affected by laser absorption because a detonation is an optically-thick reactive flow, at least in the experimental configuration we studied. In addition, the curvature of the detonation front can create a non-homogeneous optical path which complicates the interpretation of the PLIF images and LIF profiles extracted from them. Such an effect is not observed for the PLIF imaging with the frontal laser light orientation.

This work demonstrates that the use of the transverse laser orientation with a wide laser sheet and a known and appropriate spatial distribution of the laser energy allows to visualize reactive areas behind the detonation front. While these results show a qualitative agreement with experimental data, new experimental data are required to quantitatively validate this promising transverse laser orientation and to confirm whether this laser orientation provides useful additional information during visualization of the detonation front. 
0.2 0.6

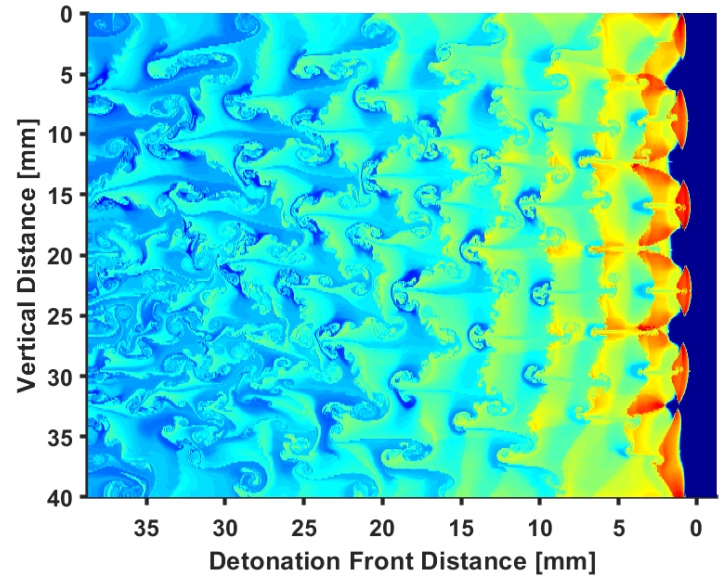

a) Normalized 2D field of $\mathrm{OH}$

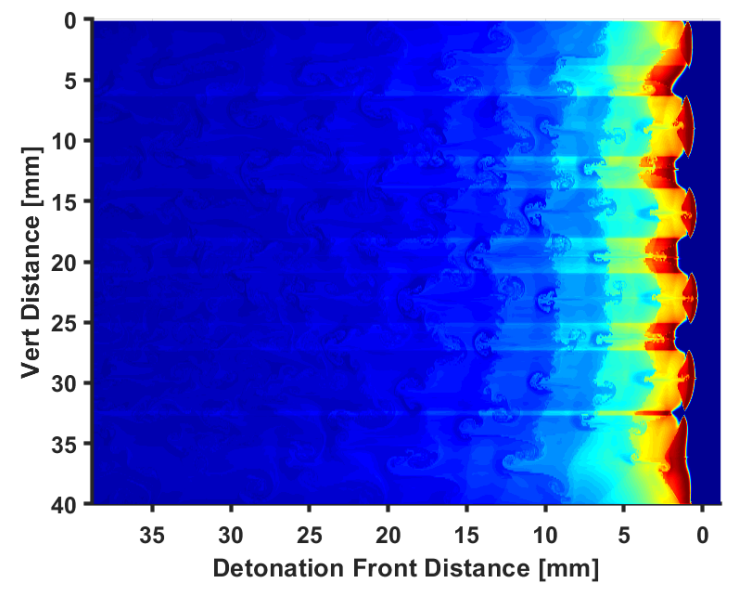

b) PLIF image with frontal laser orientation

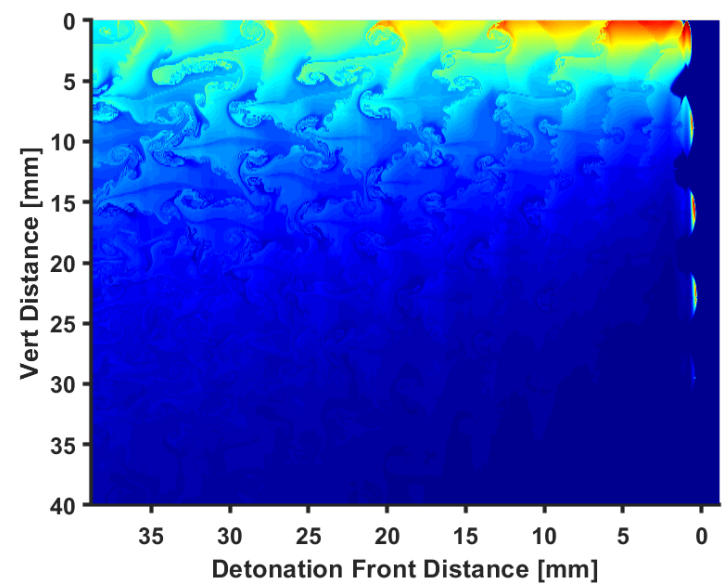

c) PLIF image with transverse laser orientation

Fig. 8 Effect of the laser sheet orientation on OH-PLIF imaging of detonation. The initial 2D field of $\mathrm{OH}$ is given in a) for comparison. Conditions similar to Wang [13]: $2 \mathrm{H}_{2^{-}}$ $\mathrm{O}_{2}-10 \mathrm{Ar}$ at $P_{1}=45.3 \mathrm{kPa}$ and $T_{1}=295 \mathrm{~K}$
Acknowledgements Research reported in this publication was supported by the King Abdullah University of Science and Technology (KAUST). RM was supported by the 1000 Young Talent of China program, a start-up fund of the Center for Combustion Energy of Tsinghua University, and the 1000 Young Talent Matching Fund from Tsinghua University. The computations were performed using HPC resources from the cluster of Pprime, the Mésocentre de Calcul Poitevin and from GENCI-CINES (Grant 2018-A0052B07735). This work was supported by the CPER FEDER Project of Région Nouvelle Aquitaine. The authors are grateful to Joanna M. Austin for allowing the use of her experimental OH-PLIF image in Fig. 3.

\section{Conflict of interest}

The authors declare that they have no conflict of interest.

\section{References}

1. Austin, J.M.: The role of instability in gaseous detonation. Ph.D, California Institute of Technology (2003)

2. Pintgen, F.: Laser-optical visualization of detonation structures, Master thesis, Technische Universität München (2000)

3. Pintgen, F., Eckett, C.A., Austin, J.M., Shepherd, J.E.: Direct observations of reaction zone structure in propagating detonations. Combust. Flame 133(3), 211229 (2003). https://doi.org/10.1016/S0010-2180(02) 00458-3

4. Austin, J.M., Pintgen, F., Shepherd, J.E.: Reaction zones in highly unstable detonations. Proceedings of the Combustion Institute 30(2), 1849-1857 (2005) https://doi. org/10.1016/j.proci.2004.08.157

5. Li, L., Li, J.M., van Nguyen, B., Teo, C.J., Chang, P.H. Khoo, B.C.: A study of detonation re-initiation through multiple reflections in a 90-degree bifurcation channel. Combust. Flame 180, 207-216 (2017). https : //doi.org/ $10.1016 / j$.combustflame.2017.03.004

6. Li, L.: Reflected re-initiation of gaseous detonation. Ph.D, National University Of Singapore (2017)

7. Radulescu, M.I.: A detonation paradox: Why inviscid detonation simulations predict the incorrect trend for the role of instability in gaseous cellular detonations? Combust. Flame 195, 151-162 (2018). https://doi.org/10. $1016 /$ j.combustflame.2018.05.002

8. Mével, R., Davidenko, D., Austin, J.M., Pintgen, F., Shepherd, J.E: Application of a laser induced fluorescence model to the numerical simulation of detonation waves in hydrogen-oxygen-diluent mixtures. Int. J. Hydrog. Energy 39(11), 6044-6060 (2014). https://doi. org/10.1016/j.ijhydene.2014.01.182

9. Mével, R., Davidenko, D., Lafosse, F., Chaumeix, N., Dupré, G., Paillard, C.É., Shepherd, J.E.: Detonation in hydrogen-nitrous oxide-diluent mixtures: An experimental and numerical study. Combust. Flame 162(5), 1638-1649 (2015). https://doi.org/10.1016/j. combustflame.2014.11.026

10. Mével, R.: Optical regime diagram of the shock tube/pulsed laser-induced fluorescence imaging technique. Chem. Phys. Lett. 730, 283-288 (2019). https: //doi.org/10.1016/j.cplett.2019.06.009 
11. Eder, A.: Brennverhalten schallnaher und überschallschneller wasserstoff-luft flammen. Ph.D thesis, Technische Universität München (2001)

12. Lee, S.Y., Watts, J., Saretto, S., Pal, S., Conrad, C., Woodward, R., Santoro, R.: Deflagration to detonation transition processes by turbulence-generating obstacles in pulse detonation engines. J. Propul. Power 20(6), 10261036 (2004). https://doi.org/10.2514/1.11042

13. Wang, C., Xu, S., Fei, L.: Study on reaction zone structure of gaseous detonation wave by planar laser induced fluorescence technique. Chinese Journal of Theoretical and Applied Mechanics 23(5), 661-667 (2007). https: //doi.org/10.6052/0459-1879-2007-5-2005-639

14. Bessler, W.G., Schulz, C., Sick, V., Daily, J.W.: A versatile modeling tool for nitric oxide lif spectra. In: Proceedings of the Third Joint Meeting of the US Sections of The Combustion Institute, P105 (2003).

15. Hanson, R.K., Mitchell Spearrin, R., Goldenstein, C.S.: Spectroscopy and optical diagnostics for gases. Springer (2016). https://doi.org/10.1007/978-3-319-23252-2

16. Gordon, I.E., Rothman, L.S., Hill, C., Kochanov, R.V., Tan, Y., Bernath, P.F., Birk, M., Boudon, V., Campargue, A., Chance, K.V., Drouin, B.J., Flaud, J.M., Gamache, R.R., Hodges, J.T., Jacquemart, D., Perevalov, V.I., Perrin, A., Shine, K.P., Smith, M.A., Tennyson, J., Toon, G.C., Tran, H., Tyuterev, V.G., Barbe, A., Császár, A.G., Devi, V.M., Furtenbacher, T., Harrison, J.J., Hartmann, J.M., Jolly, A., Johnson, T.J., Karman, T., Kleiner, I., Kyuberis, A.A., Loos, J., Lyulin, O.M., Massie, S.T., Mikhailenko, S.N., Moazzen-Ahmadi, N., Müller, H., Naumenko, O.V., Nikitin, A.V., Polyansky, O.L., Rey, M., Rotger, M., Sharpe, S.W., Sung, K., Starikova, E., Tashkun, S.A., Auwera, J.V., Wagner, G., Wilzewski, J., Wcisło, P., Yu, S., Zak, E.J.: The HITRAN2016 molecular spectroscopic database. J. Quant. Spectrosc. Radiat. Transf. 203, 3-69 (2017). https:// doi.org/10.1016/j.jqsrt.2017.06.038

17. Thiery, L., Prenel, J.P., Porcar, R.: Theoretical and experimental intensity analysis of laser light sheets for flow visualization. Opt. Commun. 123:801-809 (1996). https://doi.org/10.1016/0030-4018(95)00523-4

18. Mével, R., Javoy, S., Lafosse, F., Chaumeix, N., Dupré, G., Paillard, C.E.: Hydrogen-nitrous oxide delay times: Shock tube experimental study and kinetic modelling. Proceedings of the Combustion Institute 32(1), 359366 (2009). https://doi.org/10.1016/j.proci. 2008. 06.171

19. Mével, R., Sabard, J., Lei, J., Chaumeix, N.: Fundamental combustion properties of oxygen enriched hydrogen/air mixtures relevant to safety analysis: Experimental and simulation study. Int. J. Hydrog. Energy 41(16), 6905-6916 (2016). https://doi.org/10.1016/j. ijhydene.2016.03.026

20. Davidenko, D., Mével, R., Dupré, G.: Numerical study of the detonation structure in rich $\mathrm{H}_{2}-\mathrm{NO}_{2} / \mathrm{N}_{2} \mathrm{O}_{4}$ and very lean $\mathrm{H}_{2}-\mathrm{N}_{2} \mathrm{O}$ mixtures. Shock Waves 21(2):85-99 (2011). https://doi.org/10.1007/s00193-011-0297-z

21. Gallier, S., Le Palud, F., Pintgen, F., Mével, R., Shepherd, J.E.: Detonation wave diffraction in $\mathrm{H}_{2}-\mathrm{O}_{2}$ - Ar mixtures. Proceedings of the Combustion Institute 36(2), 2781-2789 (2017). https://doi.org/10.1016/j.proci. 2016.06 .090 .

22. Shepherd, J.E.: Chemical kinetics of hydrogen-air-diluent detonations. Prog. Astronaut. Aeronaut. 106, 263-293 (1986).

23. Browne, S., Zeigler, J., Shepherd,J.E.: Numerical solution methods for shock and detonation jump conditions. Tech- nical report FM2006-006, Graduate Aeronautical Laboratories California Institute of Technology (2008).

24. Mével, R.: Etude de mécanismes cinétiques et des propriétés explosives des systèmes hydrogène-protoxyde d'azote et silane-protoxyde d'azote : application à la sécurité industrielle. Ph.D thesis (2009). http://www. theses.fr/20090RLE2068

25. Mével, R., Gallier, S.: Structure of detonation propagating in lean and rich dimethyl ether-oxygen mixtures. Shock Waves 28(5), 955-966 (2018). https://doi.org/ $10.1007 / \mathrm{s} 00193-018-0837-\mathrm{x}$

26. Reynaud, M., Virot, F., Chinnayya, A.: A computational study of the interaction of gaseous detonations with a compressible layer. Phys. Fluids 29(5):056101 (2017). https://doi.org/10.1063/1.4982659

27. Taileb, S.; Melguizo-Gavilanes, J.; Chinnayya, A.: Chemistry Modeling Effects on the Interaction of a Gaseous Detonation with an Inert Layer. 27th International Colloquium on the Dynamics of Explosions and Reactive Systems, Beijing, Paper 320 (2019)

28. Savard, B., Xuan, Y., Bobbitt, B., Blanquart, G.: A computationally-efficient, semi-implicit, iterative method for the time-integration of reacting flows with stiff chemistry. J. Comput. Phys. 295, 740-769 (2015). https: //doi.org/10.1016/j.jcp.2015.04.018

29. Sow, A.; Chinnayya, A.; Hadjadj, A.: On the viscous boundary layer of weakly unstable detonations in narrow channels. Comput Fluids 30, 449-458 (2019). https: //doi.org/10.1016/j.compfluid.2018.11.006

30. Sharpe, G.J.: Transverse waves in numerical simulations of cellular detonations. J. Fluid Mech. 447, 31-51 (2001). https://doi.org/10.1017/S0022112001005535 
Appendix 1: Sequence of images of the Voigt profile evolution along the detonation front

This appendix presents a more detailed evolution of the Voigt profiles presented in Fig. 1, with a higher spatial resolution to better illustrate the absorption lineshape evolution along the distance behind the leading shock (X). Similarly to the results presented in Fig. 1, the absorption lines are simulated based on Wang's experimental conditions with a transverse laser orientation $\left(\delta_{\mathrm{D}}=0\right.$ and $\left.\delta_{\text {Tot. }}=\delta_{\mathrm{P}}\right)$. A movie presenting this sequence of images with intermediate positions is available in the Supplementary material.

\section{Appendix 2: Additional 1D validation cases with the transverse laser orientation.}

Figure 10 presents two additional $1 \mathrm{D}$ validation cases extracted from Wang's OH-PLIF image [13]. As presented for areas 1 and 2, the LIF model reproduces qualitatively the overall intensity profile for both areas 3 and 4 when the correction accounting for the saturation of the camera is included. In area 3, the fluorescence decay has an overall offset of $2 \mathrm{~mm}$, which is relatively small considering the non-ideality in the experimental image (the tilted detonation front). In area 4 , the double peak structure is reproduced in the simulation with a local fluorescence intensity minimum located near $2 \mathrm{~mm}$ as observed experimentally. The double peak intensity is underestimated by $25 \%$ in area 4 , this might indicate that the fluorescence signal attenuation is overestimated in the current model or this might be due to an overestimated laser intensity attenuation. As discussed in Sec.3.1 and emphasized in the conclusions, new experimental data are required to fully validate the LIF model for the transverse laser orientation.

\section{Appendix 3: Relevance of the new LIF model}

This appendix demonstrates the need to develop the new LIF model and to consider the improvements presented in this work, notably the absorption line shifts and the Voigt profile calculation. As explained in Sec.3.2, the two laser orientations (the transverse and the frontal) must be considered independently as the Doppler shift is significantly different for these two orientations. Only three characteristic distances from the detonation front are presented in Figs. 11 and 12: von Neumann state $\left(X_{\mathrm{vN}}\right)$, induction length $\left(\Delta_{i}\right)$, CJ state $\left(X_{\mathrm{CJ}}\right)$.

Figure 11 emphasizes the contribution of the Doppler shift $\left(\delta_{\mathrm{D}}\right)$ for Wang's conditions, for both the two and the five line calculations of the Voigt profile. As the laser linecenter is constant, the $\delta_{\mathrm{D}}$ contribution is observed by comparing the shift of the Voigt profile maximum with the laser linecenter between Figs. 11a and 11b for the three positions. As the $\delta_{\mathrm{D}}$ decreases with the distance behind the leading shock and is mainly contributing to the $\delta_{\text {Tot. }}$, it must be noted that the shift is the strongest for $X_{\mathrm{vN}}$.

Figure 12 presents the evolution of both the two and the five line calculation of the Voigt profile at two different pressure conditions using a frontal laser orientation and for the three distances from the detonation front, defined previously: $X_{\mathrm{vN}}, \Delta_{i}$, and $X_{\mathrm{CJ}}$.

These figures show the need to consider the contribution of additional absorption lines for higher initial pressure in this specific spectral range. 

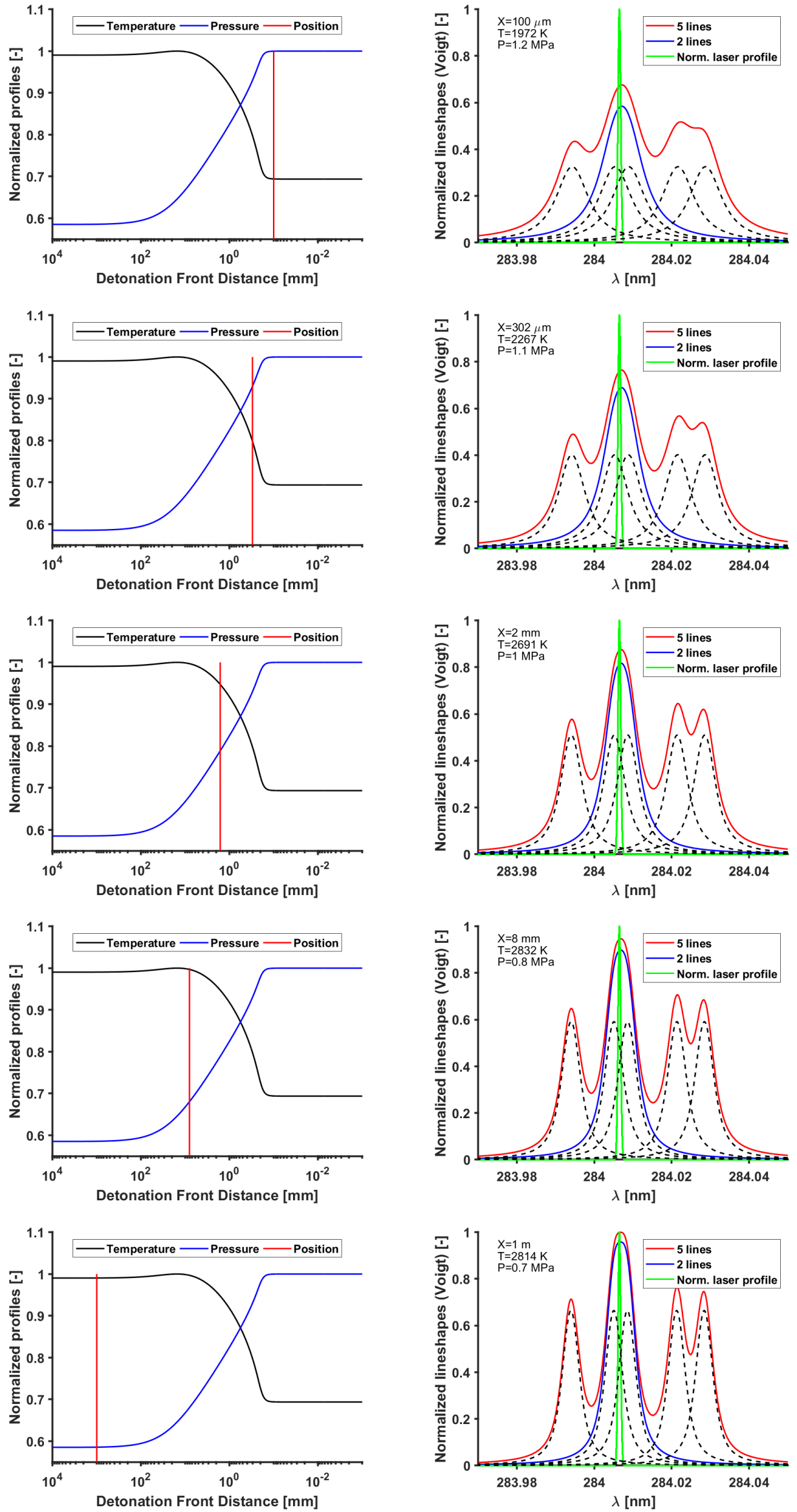

Fig. 9 High resolution spatial evolution of the transitions lineshape as a function of the distance relative to the detonation front. For each sequence, the vertical red line on the left figure represents the actual distance relative to the detonation front that is used to calculate the transitions lineshape on the right 


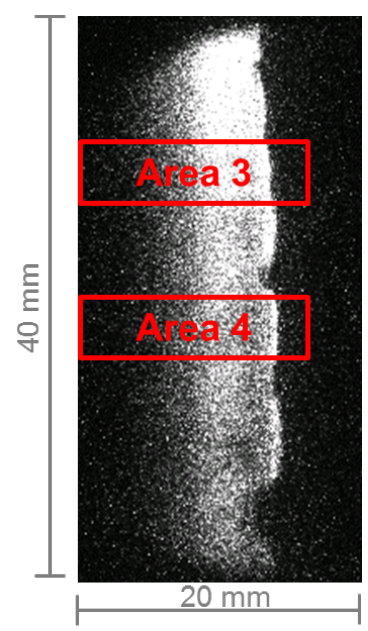

a) Experimental OH-PLIF

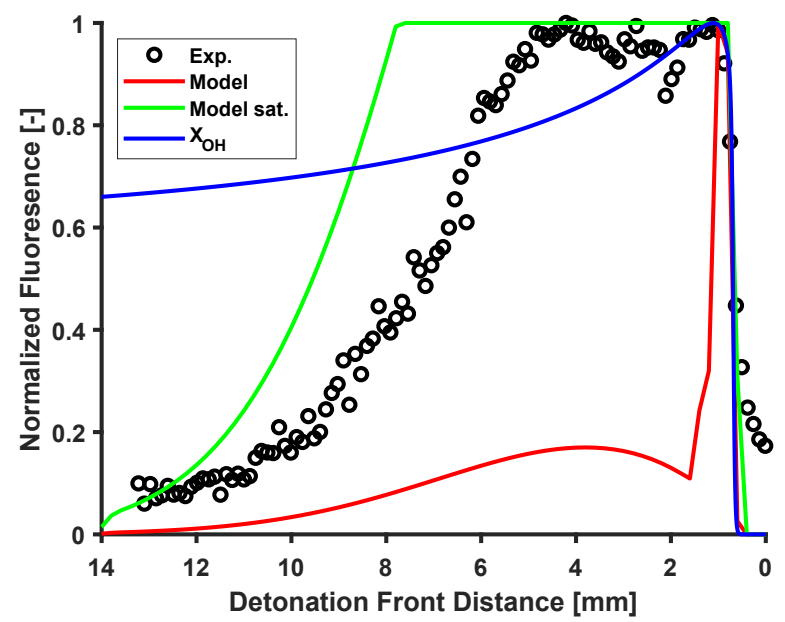

b) Validation on area 3

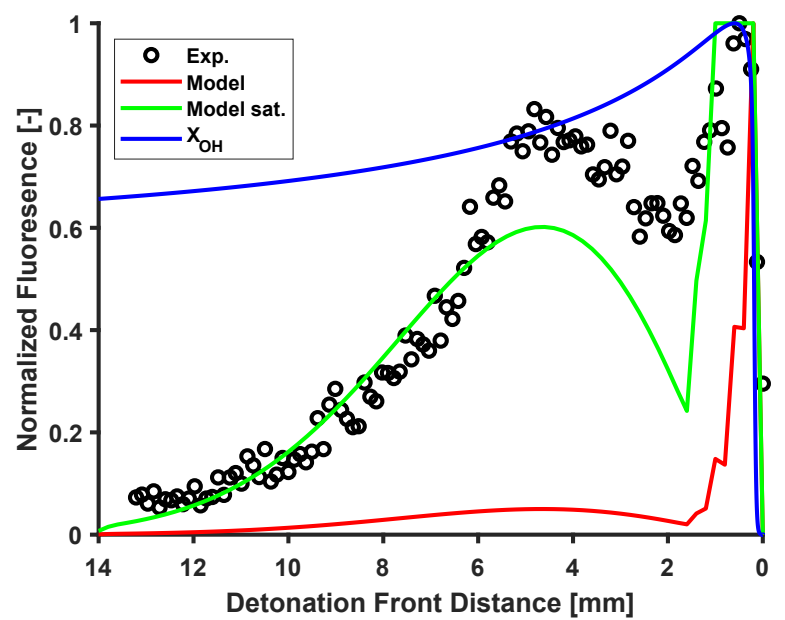

c) Validation on area 4

Fig. 10 1D validation of the transverse laser orientation using an OH-PLIF image from Wang [13], in a). 1D experimental (black symbols) and ZND (red and green lines) LIF profiles, obtained for area 3 and 4, are respectively in b) and c). ZND OH mole fractions (blue lines) are represented for comparison. Conditions: $2 \mathrm{H}_{2}-\mathrm{O}_{2}-10 \mathrm{Ar}$ at $P_{1}=45.3 \mathrm{kPa}$ and $T_{1}=295 \mathrm{~K}$ 

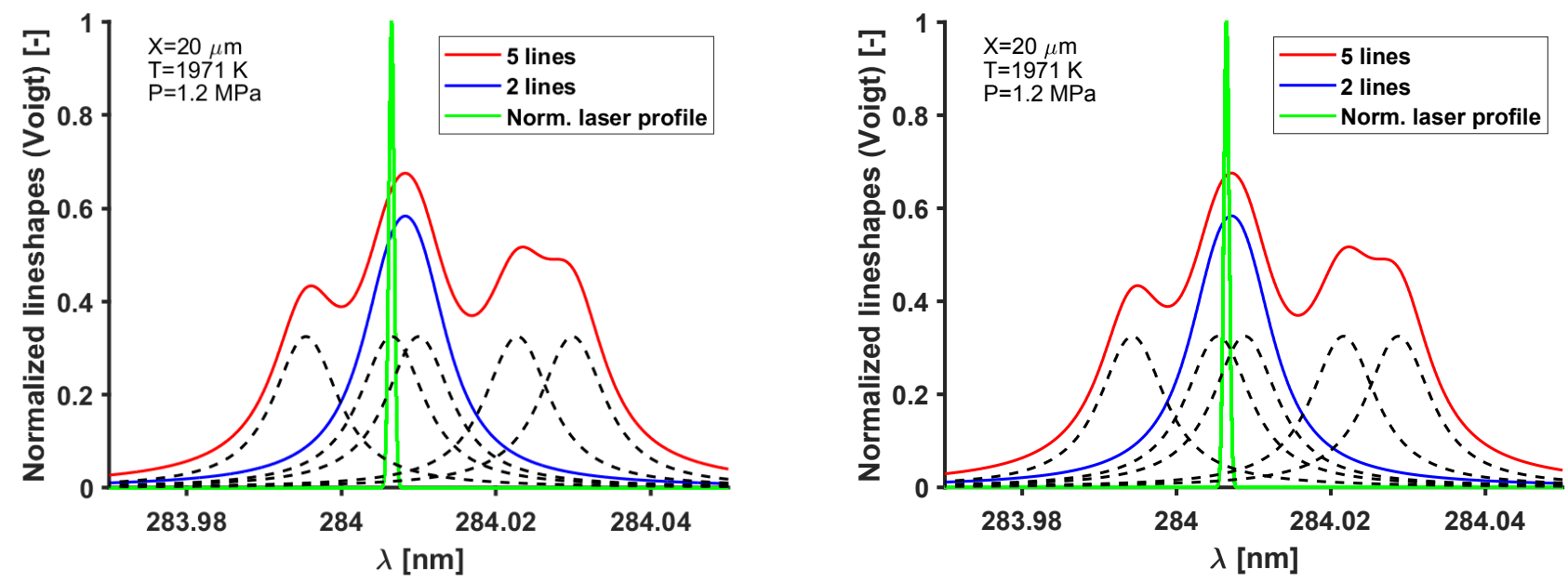

i) $X_{\mathrm{vN}}$
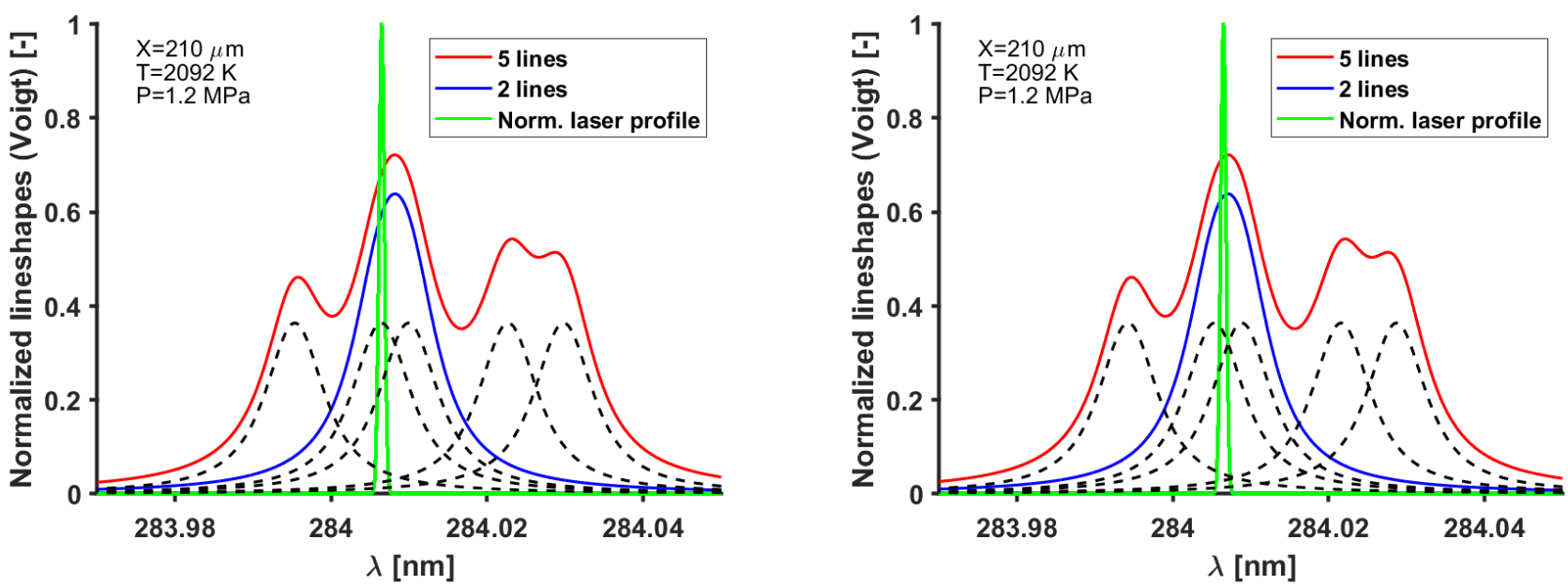

ii) $\Delta_{i}$
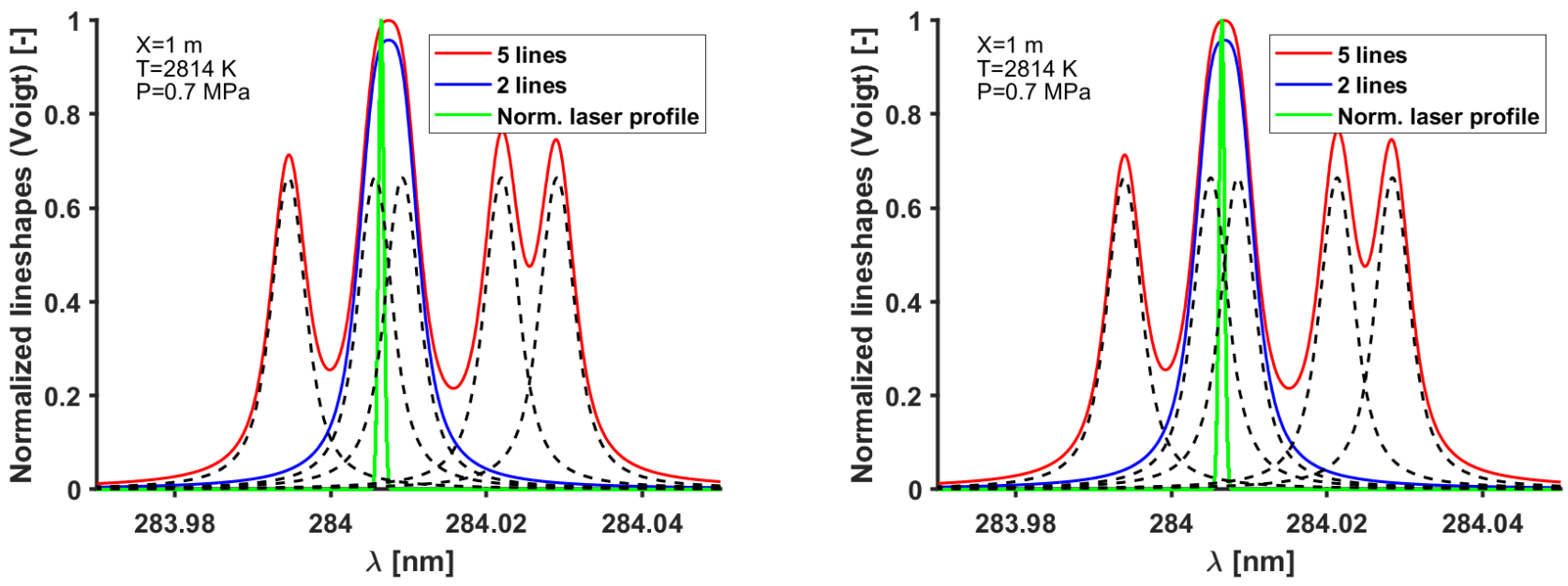

a) Frontal laser orientation

iii) $X_{\mathrm{CJ}}$

b) Transverse laser orientation

Fig. 11 Effect of the laser sheet orientation on the absorption line shifts in Wang's conditions [13]. Conditions: $2 \mathrm{H}_{2}-\mathrm{O}_{2}-10 \mathrm{Ar}$ at $P_{1}=45.3 \mathrm{kPa}$ and $T_{1}=295 \mathrm{~K}$ 

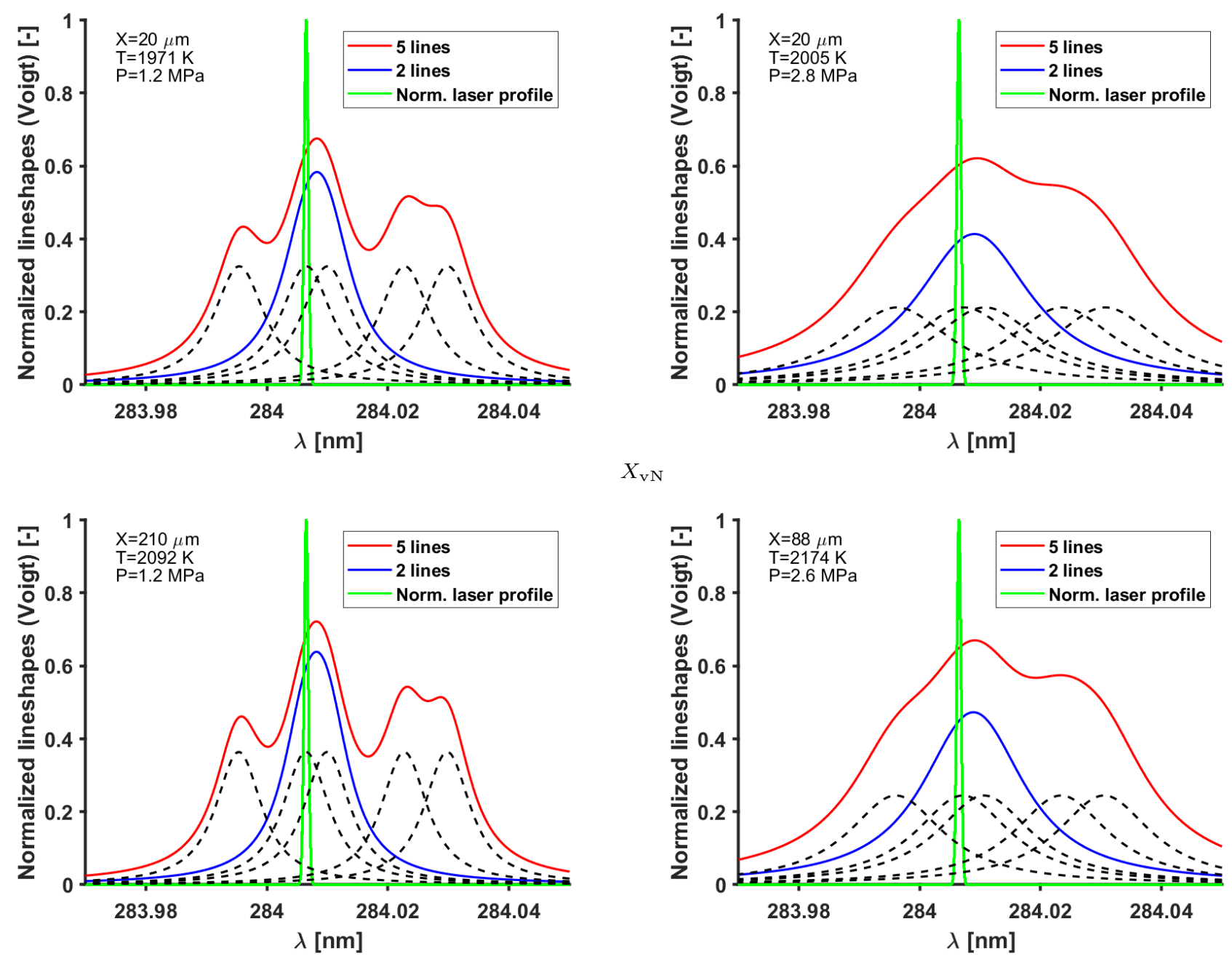

$\Delta_{i}$
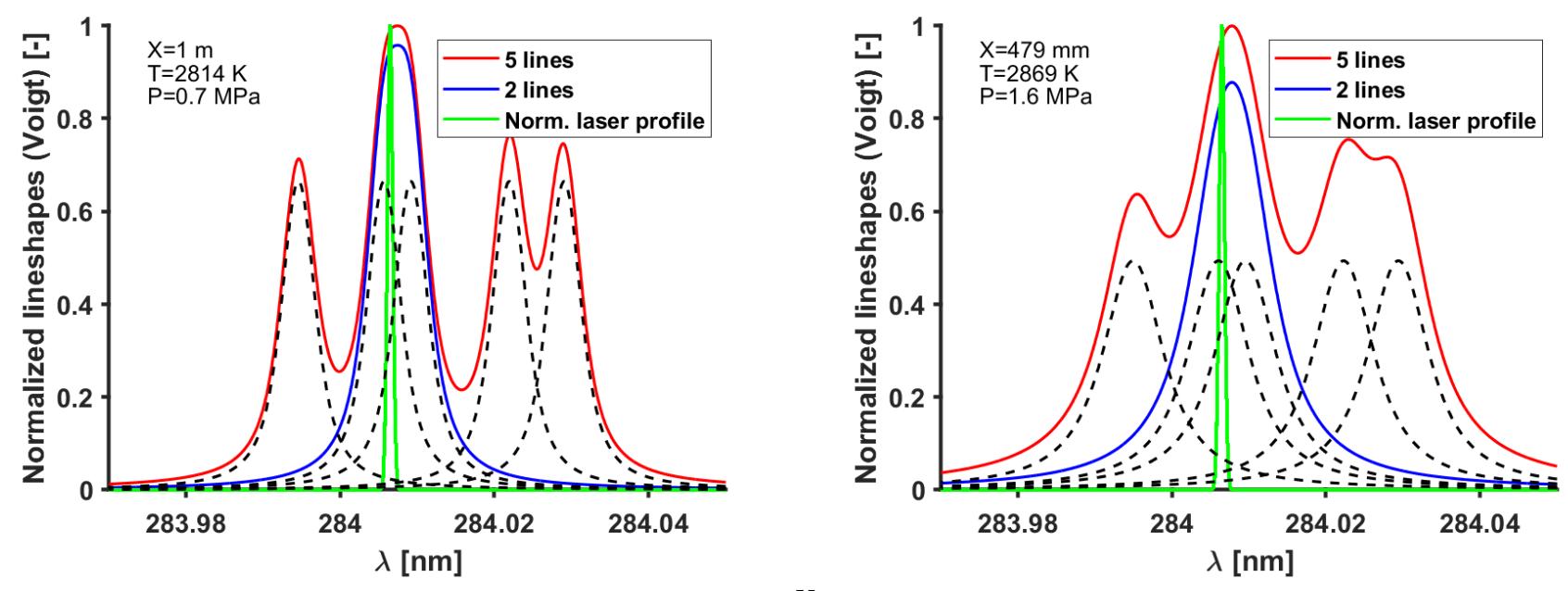

a) Wang's conditions

b) Wang's mixture with $P_{1}=100 \mathrm{kPa}$

Fig. 12 Effect of the initial pressure on the absorption lineshapes in Wang's experimental conditions [13] with a frontal laser orientation. Conditions: $2 \mathrm{H}_{2}-\mathrm{O}_{2}-10 \mathrm{Ar}$ at $T_{1}=295 \mathrm{~K}$ and both $P_{1}=45.3 \mathrm{kPa}$ and $P_{1}=100 \mathrm{kPa}$, respectively in a) and b) 